\title{
ASPECTOS ELETROMIOGRÁFICOS PREDITORES DA EVOLUÇÃO MOTORA DE PACIENTES COM NEUROESQUISTOSSOMOSE
}

\author{
Carolina da Cunha Correia', Luiz Ataíde Júnior², \\ Otávio Gomes Lins', Maria Lúcia Brito Ferreira ${ }^{4}$
}

\begin{abstract}
RESUMO - A neuroesquistossomose na forma mielorradicular é freqüentemente observada nos pacientes que residem no Nordeste do Brasil. Apesar disso, a evolução dos seus distúrbios neurológicos e padrões eletromiográficos são pouco estudados nesse grupo de pacientes. O objetivo deste estudo foi descrever e comparar a evolução motora com as anormalidades eletromiográficas de pacientes com neuroesquistossomose. Foram realizadas eletromiografias dos membros inferiores em 21 pacientes com diagnóstico presuntivo de radiculomielite esquistossomótica. O padrão eletromiográfico de $95,2 \%$ dos casos foi de multirradiculopatia axonal lombo-sacra, havendo extensão variável de desnervação, com predomínio de $L_{2}$ a $S_{2}$. Foram identificados graus variáveis de paraparesia, havendo evolução motora mais favorável nos pacientes com envolvimento de menor número de raízes. A eletromiografia poderá fornecer dados prognósticos da evolução motora dos pacientes com radiculomielite esquistossomótica.
\end{abstract}

PALAVRAS-CHAVE: neuroesquistossomose, eletromiografia, prognóstico.

\begin{abstract}
Electromyografic patterns predictive of motor evolution in neuroschistosomiasis
ABSTRACT - Neuroschistosomiasis in myeloradicular pattern is frequently observed in patients from Northeast of Brazil. Despite of this, the evolution of neurologic and electromyografic patterns is not well studied in this group of patients. The aims of this study were to describe and compare the clinic and electromyografic abnormalities of patients with neuroschistosomiasis and radicular involvement. We analyzed 21 electromyographic exams of the lower limbs carried out in the initial presentation of the disease. Electromyographic pattern of $95.2 \%$ of patients was compatible to axonal lombosacral multirradiculopathy, with variable denervation extension, but predominanthy from levels $L_{2}$ to $S_{2}$. There was variable degree of deficit in the lower limbs, and the follow-up of motor disturbances was better more frequently when the roots were involved in smaller number. Electromyography should permit to know the motor prognosis of patients with myeloradiculitis due schistosomiasis.
\end{abstract}

KEY WORDS: neuroschistosomiasis, electromyography, diagnosis.

Do ponto de vista epidemiológico, a esquistossomose mansônica acomete cerca de $10 \%$ da população brasileira, estimando-se haver 17 milhões de esquistossomóticos no Brasil'1. Segundo a Organização Mundial de Saúde, a maioria dos dados clínicos e laboratoriais, relativos ao Schistosoma mansoni, provêm do

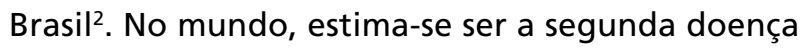
parasitária de maior incidência, sendo sobrepujada apenas pela malária ${ }^{3,4}$. As formas de acometimento neurológico, na infestação pelo $S$. mansoni, são classificadas de maneira não uniforme na literatura ${ }^{5-7}$. Em diversos estudos foi adotada a classificação de Peregrino, cujas formas e freqüências são: mielorradicu- lítica (44\%), mielítica $(29,4 \%)$, pseudotumoral $(24,7 \%)$, além da forma radiculítica pura e da síndrome da artéria espinhal anterior, relatadas com menor freqüência na literatura ${ }^{8}$.

O diagnóstico de certeza da neuroesquistossomose baseia-se na presença de ovos do parasito em biópsia medular, porém, por ser método invasivo, não é realizado de rotina. Dada a necessidade de critérios para o diagnóstico presuntivo da esquistossomose medular, o Centers for Disease Control and Prevention (CDC) estabeleceu como parâmetros, a presença de sintomas neurológicos envolvendo a porção inferior da medula torácica e superior da lombar,

\footnotetext{
${ }^{1}$ Médica Neurologista, Doutoranda do Departamento de Doenças Infecto-Parasitárias, Universidade Federal de Pernambuco, Recife PE, Brasil (UFPE); ${ }^{2}$ Professor Adjunto da Disciplina de Neurologia, Universidade de Pernambuco, Recife PE, Brasil (UPE) e UFPE; ${ }^{3}$ Neurofisiologista, Recife PE, Brasil; ${ }^{4}$ Neurologista, Chefe do Serviço de Neurologia do Hospital da Restauração, Recife PE, Brasil.
}

Recebido 4 Julho 2006, recebido na forma final 21 Setembro 2006. Aceito 7 Novembro 2006. 
além da demonstração da infestação pelo Schistosoma por método complementar, microscópico ou sorológico, associada à exclusão de outras causas de mielite transversa ${ }^{9,10}$. No entanto, embora seja inquestionável a contribuição desses exames para o diagnóstico da neuroesquistossomose, não há método que forneça alterações patognomônicas da doença.

$\mathrm{Na}$ busca de aprimorar o diagnóstico clínico da doença e melhor entendimento de sua evolução, são fundamentais as pesquisas epidemiológicas e de novos exames. Apesar das incapacidades motora, sensitiva e esfincteriana que compõem o quadro clínico da doença, poucos estudos abordam aspectos prognósticos da neuroesquistossomose, sendo mais escassos ainda os que utilizam a avaliação eletromiográfica (EMG) nestes pacientes ${ }^{4-6,11-13}$. Na observação clínica cotidiana, foi levantada a possibilidade de a extensão do comprometimento periférico, associada ao quadro central, ser um fator determinante de mau prognóstico na evolução destes pacientes. A EMG pode permitir que se identifique tal comprometimento, mesmo em pacientes nos quais este esteja ausente ao exame neurológico clínico inicial ${ }^{6}$.

Sendo assim, objetivou-se descrever os aspectos eletromiográficos iniciais dos pacientes com neuroesquistossomose mansônica, comparando-os com os dados de evolução motora.

\section{MÉTODO}

Foram realizadas 21 eletromiografias dos membros inferiores em pacientes com diagnóstico presuntivo de neuroesquistossomose firmado segundo critérios do $\mathrm{CDC}^{9,10}$, atendidos no Hospital da Restauração, Recife (PE), no período de junho de 2000 a junho de 2003.

Os parâmetros de força muscular foram obtidos segundo graduação estipulada pelo Medical Research Council (MRC). Para o grupo de pacientes com incapacidade de deambular ou que deambulavam com apoio foi pontuado escore do MRC $\leq 3$ pontos; aqueles que tiveram deambulação preservada receberam escore $\geq 4$.

As eletromiografias dos membros inferiores foram realizadas por meio do aparelho Medelec Synergy Notebook, após tempo médio de $18 \pm 2$ dias da instalação do quadro neurológico. No estudo da condução nervosa, foram empregados eletrodos descartáveis de superfície, obedecendo aos critérios da Associação Americana de Medicina Eletrodiagnóstica, mantida a temperatura dos membros inferiores superior a $34^{\circ} \mathrm{C}$, durante o exame. Foram avaliadas as amplitudes dos potenciais e velocidades de condução dos nervos tibiais, fibulares comuns e surais. $\mathrm{Na}$ avaliação dos potenciais de nervos sensitivos, foi utilizada técnica antidrômi$\mathrm{Ca}^{14}$. A amplitude de todos os potenciais foi avaliada da base ao pico. As latências mínimas das respostas tardias (onda $\mathrm{F}$ dos nervos tibiais e reflexos $\mathrm{H}$ ) também foram aferidas, considerando-se sua variabilidade de acordo com a estatura do paciente $\mathrm{i}^{15,16}$.
Na eletromiografia foram avaliados músculos de acordo com nível miotômico, incluindo componentes de $L_{2}-L_{3}-L_{4}-$ $\mathrm{L}_{5}-\mathrm{S}_{1}-\mathrm{S}_{2}$ bilateralmente, além de musculatura paravertebral lombo-sacra. Analisaram-se a presença de atividade espontânea, além do padrão de recrutamento e configuração dos potenciais de unidades motoras. Na pesquisa de atividade espontânea, utilizou-se sensibilidade de $100 \mu \mathrm{V} /$ divisão e, na análise dos potenciais de unidades motoras, $1 \mathrm{mV} /$ divisão, com velocidade de varredura de $10 \mathrm{~ms} /$ divisão e filtros passa-faixa com valores entre $2 \mathrm{~Hz}$ a $10 \mathrm{KHz}^{16-18}$.

Este estudo foi aprovado pelo Comitê de Ética em Pesquisa do Hospital da Restauração, sob número 038/03.

\section{RESULTADOS}

Todos os pacientes avaliados tiveram apresentação clínica da forma mielorradiculítica da neuroesquistossomose. Do ponto de vista do déficit motor inicial, $20(95,2 \%)$ pacientes apresentaram paraparesia de graus variados, enquanto que um paciente era paraplégico à admissão.

$\mathrm{Na}$ etapa da condução nervosa, observou-se que a amplitude dos potenciais e a velocidade de condução foram normais em $100 \%$ dos nervos surais e na maioria dos potenciais motores dos nervos tibiais e fibulares comuns, exceção feita a dois casos, nos quais a amplitude ou a velocidade estava abolida em nervo fibular (Tabela 1).

Quanto às respostas tardias de condução do nervo tibial, identificou-se normalidade de latência mínima de onda $\mathrm{F}$ em 13 (61,9\%) e anormalidade em oito $(38,1 \%)$, consistindo de abolição em seis $(75 \%)$ casos e prolongamento em dois ( $25 \%)$. A latência mínima do reflexo $\mathrm{H}$ em nervo tibial foi normal em $11(52,4 \%)$ pacientes e elevada em $10(47,6 \%)$, dos quais nove $(90 \%)$ com resposta abolida e um $(10 \%)$, prolongada.

No estudo dos eletromiogramas, diagnosticou-se anormalidade em 20 (95,2\%) casos, consistindo em sinais de desnervação situada nos níveis de: $L_{2}$ a $L_{5}$ em $2(10 \%)$ pacientes; $L_{2}$ a $S_{2}$ em $12(60 \%), L_{5}$ a $S_{2}$ em $5(25 \%)$ e $S_{1}-S_{2}$ em 1 ( $\left.5 \%\right)$ caso. $O$ estudo eletromiográfico foi normal em um paciente.

O padrão de alterações eletromiográficas pode ser descrito como o de multirradiculopatia axonal lombo-sacra, caracterizada por amplitudes normais ou reduzidas dos potenciais de músculos dos nervos tibiais e fibulares, com velocidades normais ou discretamente reduzidas, além de potenciais de nervos sensitivos surais normais. A eletromiografia mostrou desnervação nos múltiplos miótomos lombo-sacros de $L_{2}$ a $S_{2}$, em variados níveis.

Considerando apenas os 19 pacientes $(90,4 \%)$ submetidos à eletromiografia e reavaliados clinicamente após a alta hospitalar, observamos os seguintes níveis de denervação (Tabela 2): 


\begin{tabular}{|c|c|c|c|c|c|c|}
\hline \multirow[t]{3}{*}{ Parâmetros da EMG } & \multicolumn{6}{|c|}{ Resultado } \\
\hline & \multicolumn{2}{|c|}{ Abolido } & \multicolumn{2}{|c|}{ Diminuído } & \multicolumn{2}{|c|}{ Normal } \\
\hline & $\mathrm{n}^{\circ}$ & $\%$ & $\mathrm{n}^{\circ}$ & $\%$ & $\mathrm{n}^{\circ}$ & $\%$ \\
\hline \multicolumn{7}{|l|}{ Amplitude } \\
\hline Tibial (mV) & - & - & 6 & 28,6 & 15 & 71,4 \\
\hline Fibular (mV) & 2 & 9,5 & 8 & 38,1 & 11 & 52,4 \\
\hline Sural (mV) & - & - & - & - & 21 & 100 \\
\hline \multicolumn{7}{|c|}{ Velocidade de condução (m/s) } \\
\hline Tibial & - & - & 2 & 9,5 & 19 & 90,5 \\
\hline Fibular & 1 & 4,8 & 1 & 4,8 & 19 & 90,5 \\
\hline Sural & - & - & 1 & 4,8 & 20 & 95,2 \\
\hline
\end{tabular}

Tabela 2. Distribuição de 19 pacientes submetidos à eletromiografia e à reavaliação segundo nível acometido e evolução dos distúrbios motores.

\begin{tabular}{|c|c|c|c|c|c|c|c|c|}
\hline \multirow{3}{*}{$\begin{array}{c}\text { Nível } \\
\text { acometido }\end{array}$} & \multicolumn{6}{|c|}{ Resultado ao final de tempo mediano de três meses } & \multicolumn{2}{|c|}{ Total } \\
\hline & \multicolumn{2}{|c|}{ Incapacidade de deambular } & \multicolumn{2}{|c|}{ Deambulação com apoio } & \multicolumn{2}{|c|}{ Deambulação sem apoio } & \multirow[b]{2}{*}{$\mathrm{n}$} & \multirow[b]{2}{*}{$\%$} \\
\hline & $\mathrm{n}$ & $\%$ & $\mathrm{n}$ & $\%$ & $\mathrm{n}$ & $\%$ & & \\
\hline L2 - L5 & - & - & 1 & 14,3 & 1 & 11,1 & 2 & 10,5 \\
\hline L2 - S2 & 3 & 100 & 5 & 71,4 & 3 & 33,3 & 11 & 57,9 \\
\hline L5 - S2 & - & - & - & - & 5 & 55,6 & 5 & 26,3 \\
\hline $\mathrm{S1} 1-\mathrm{S} 2$ & - & - & 1 & 14,3 & - & - & 1 & 5,3 \\
\hline Total & 3 & 100 & 7 & 100 & 9 & 100 & 19 & 100 \\
\hline
\end{tabular}

- Dentre os seis $(31,6 \%)$ pacientes com comprometimento motor de $L_{5}$ a $S_{2}$ ou $S_{1}-S_{2}$, cinco $(83,3 \%)$ evoluíram com recuperação da função motora, independentemente do grau de comprometimento inicial, e um $(16,7 \%)$ permaneceu deambulando com apoio;

- Os $11(57,8 \%)$ pacientes com comprometimento motor extenso, de $\mathrm{L}_{2}$ a $\mathrm{S}_{2}$, mostraram tendência de evolução menos favorável: oito $(72,7 \%)$ mantiveram-se incapazes de deambular ou permaneceram com acentuado déficit motor, dependente de apoio para deambulação ;

- Os dois pacientes com comprometimento ao nível de $L_{2}$ a $L_{5}$ deambulavam quando do exame reavaliatório.

\section{DISCUSSÃO}

Poucos estudos abordam a presença de alterações eletromiográficas em pacientes com neuroesquistossomose mansônica, 12,19. O primeiro, datado de 1972, relata padrão de polineuropatia ${ }^{19}$. Em 1977, Lechtenberg e Vaida descrevem a forma medular da neuroesquistossomose em quatro pacientes, tendo realizado eletromiografia em apenas dois destes. Sem tecer comentários adicionais sobre a utilidade dos achados, os autores mencionam desnervação abaixo do nível $\mathrm{L}_{3}$ em um dos casos e denervação parcial em $\mathrm{L}_{4}-\mathrm{L}_{5}-\mathrm{S}_{1}$ com evidência de reinervação no outro paciente, tendo ambos alterações que denotavam comprometimento radicular ${ }^{12}$. Em 2002, Peregrino et al. concluem que, apesar da eletromiografia ser método sem especificidade para os casos de esquistossomose medular, pode ajudar na determinação do diagnóstico presuntivo da doença ${ }^{13}$.

A investigação diagnóstica por imagem, do envolvimento mielorradicular na esquistossomose, apesar de factível, apresenta limitações. A eletromiografia tem a vantagem de poder detectar as alterações radiculares, detalhando seu padrão de comprometimento e sua freqüência, como também de oferecer dados prognósticos, com base na hipótese de que tais alterações poderiam estar relacionadas à evolução motora desses casos.

O predomínio de normalidade das amplitudes dos potenciais sensitivos dos nervos surais foi o primeiro indicativo da possibilidade de uma lesão radicular, a 
qual se confirmou com a constatação de pouca ou nenhuma alteração na condução motora dos nervos fibulares comuns e tibiais, associada às anormalidades detectadas por meio da avaliação eletromiográfica.

As alterações de eletromiografia com padrão de desnervação foram importantes, pois refletiram degeneração axonal, sendo, portanto, sinais de pior prognóstico. Além disso, tal padrão configurou quadro multirradicular axonal bilateral, acometendo as raízes $L_{2}, L_{3}, L_{4}, L_{5}, S_{1}, S_{2}$, cuja extensão de grau variável assemelhou-se aos achados de Peregrino et al. ${ }^{13}$.

A normalidade da latência mínima da onda $F$ concordou com os resultados de Peregrino et al., porém o reflexo $\mathrm{H}$ esteve alterado menos freqüentemente do que relatado por esses autores ${ }^{13}$. Embora esses dois parâmetros não sejam exclusivos para a determinação do diagnóstico de comprometimento radicular, quando alterados, podem reforçar tal hipótese.

Abordando outras patologias, Misra e Kalita relatam os aspectos prognósticos dos resultados da eletromiografia, realizada em 17 pacientes com envolvimento radicular. Descrevem alterações em 11 casos, consistindo em presença de fibrilações ou ondas positivas em graus variáveis. No seguimento clínico, identificam que os pacientes com desnervação nos membros inferiores, presente na fase aguda, têm prognóstico ruim, ao passo que aqueles, na ausência desses achados, recuperam-se bem ${ }^{20}$.

A história natural da neuroesquistossomose não é completamente conhecida, conforme chama a atenção Ferrari et al. ${ }^{11} \mathrm{em}$ artigo de revisão, haja visto ter encontrado, não raramente, casos com período de recuperação curto (em semanas), mas também quadros com período longo de remissão. A autora comenta que, apesar de ter analisado artigos publicados em um período de 66 anos, a variabilidade do tempo de seguimento dos pacientes, de dias ou até aproximadamente três anos, além da omissão desse dado, se constituem em fatores limitantes da definição do prognóstico da doença. Apesar dessas limitações, a autora conclui que o prognóstico depende em parte de fatores ligados à própria doença, como tipo, localização e extensão da lesão, bem como à instituição precoce de terapêutica ${ }^{11}$. Alguns trabalhos tipo série de casos, predominantemente de autores nacionais, traçaram um perfil da evolução do quadro clínico. Em um deles, há informações de melhora em cerca de $62,5 \%$ dos pacientes dentre 56 estudados, porém o detalhamento dos aspectos da melhora é omitido 5 .

$\mathrm{Na}$ avaliação clínica associada à eletromiografia, detectou-se que a extensão e o nível de comprome- timento periférico inicial pareceram ter se comportado como preditores da evolução. Quando o comprometimento foi extenso, envolvendo raízes de $L_{2}$ a $\mathrm{S}_{2}$, a recuperação tendeu a ser incompleta; no entanto, para os pacientes com comprometimento de menor número de raízes, a evolução foi mais favorável.

A falta de dados comparativos, bem como o pequeno número de pacientes submetidos à eletromiografia dificultaram a análise dos resultados; mesmo assim entende-se que os achados obtidos podem ser indicativos de uma provável relação causal. Esta hipótese merece novas pesquisas, com casuística maior e seguimento sistematizado, que inclua a realização de estudo eletromiográfico quando da estabilização do quadro neurológico final. Assim será possível analisar o valor das manifestações motoras inaugurais à luz da clínica e da eletromiografia, que poderá se tornar um instrumento importante de avaliação do prognóstico.

\section{REFERÊNCIAS}

1. Matas SLA. Neuroesquistossomose. Rev Neurociências 2001;9:27-31.

2. WHO. Report of the WHO informal consultation on schistosomiasis control. WHO/CDS/CPC/SIP/99.2, 1998:16.

3. Gellido CL, Onesti S, Liena J, Suarez M. Spinal schistosomiasis. Neurology 2000;54:527.

4. Fiore M, Aloe L. Neuroinflammatory implication of Schistosoma mansoni infection in the mouse. Arch Physiol Biochem 2001;109:361-364.

5. Santos EC, Campos GB, Diniz AC, Leal JC, Rocha MOC. Perfil clínico e critérios diagnósticos da mielorradiculopatia esquistossomótica. Arq Neuropsiquiatr 2001;59:772-777.

6. Peregrino AJP, Puglia PMK, Nóbrega JPS, Livramento JA, MarquesDias MJ, Scaff M. Esquistossomose medular: análise de 80 casos. Arq Neuropsiquiatr 2002;60:603-608.

7. Valença MM. Critérios diagnósticos da esquistossomose mansônica meningomielorradicular. Arq Neuropsiquiatr 2002;60:181-182.

8. Scrimgeour EM, Gadjusek C. Involvement of the central nervous system in Schistosoma mansoni and Schistosoma haematobium infection: a review. Brain 1985;108:1023-1038.

9. Nobre V, Silva LCS, Ribas JG, et al. Schistosomal myeloradiculopathy due to Schistosoma mansoni: report on 23 cases. Mem Inst Oswaldo Cruz 2001;96:137-141.

10. Centers for Disease Control and Prevention (CDC). Acute schistosomiasis with transverse myelitis in american students returning from Kenya. MMWR Weekly 1984;33:445-447.

11. Ferrari TCA. Spinal cord schistosomiasis: a report of 2 cases and review emphasizing clinical aspects. Medicine 1999;78:176-190.

12. Lechtenberg R, Vaida GA. Schistosomiasis of the spinal cord. Neurology 1977;27:55-59.

13. Peregrino AJP, Puglia PMK, Bacheschi LA, et al. Diagnóstico da esquistossomose medular. Contribuição da ressonância magnética e eletroneuromiografia. Arq Neuropsiquiatr 2002;60:1-11.

14. Cruz MW, Scola RH, Coelho RLA. Projeto diretrizes: eletroneuromiografia na abordagem diagnóstica das neuropatias periféricas. Assoc Med Bras e Conselho Federal de Medicina 2001:1-8.

15. Liveson JA. Late responses. In Laboratory reference for clinical neurophysiology. Philadelphia 1992;237-262.

16. Kimura J. Electrodiagnosis in diseases of nerve and muscle: principles and practice. New York: Oxford University Press 2001:439-494.

17. Dillingham $\mathrm{T}$, Dasher KJ. The lumbosacral electromyographic screen: revisiting a classic paper. Clin Neurophysiol 2000;111:2219-2222.

18. Denny-Brown D, Pennybacker JB. Fibrillation and fasciculation in voluntary muscle. Brain 1938;61:311-334.

19. Mostafa M, Habib MA, Abdel-Moneim S, et al. Peripheral polyneuropathy in bilharziasis. J Egypt Med Assoc 1972;55:44-59.

20. Misra UK, Kalita J. Can electromyography predict the prognosis of transverse myelitis? J Neurol 1998;245:741-744. 